\title{
Analysis of the Argumentative Effect of Evaluative Semantics in Natural Language
}

\author{
Serge V. Gavenko \\ Moscow State Linguistic University \\ Usacheva St., 62-510, Moscow 119048 Russia \\ serge.gavenko@mtu-net.ru
}

\begin{abstract}
The article deals with the semantic aspect of the argumentative discourse. Argumentation has long been studied in the realm of the rhetorical science, though a research into the functioning of linguistic means on which any verbal human interaction is based can present to us some subtle mechanisms that subconsciously govern the process of persuasion and decision-making. The particular research reflected in this paper tries to cast some light upon argumentative power of evaluative semantics. A person who has to make a decision about something, attaches a certain value to it; by building argumentative discourse while employing and operating evaluative notions, authors are able to lead addressees to a certain desired attitude. The highest appeal have sublimated (ethical, aesthetical, emotive) evaluations, though evaluations, based on perceptive sensations may be propelled to have sublimated charge. Such a mechanism is the major point of investigation in the present paper.
\end{abstract}

\section{Argumentation as a Multi-discipline Object}

The present research is based on the analysis of evaluative semantics derived from the notion of sense perception used in argumentative discourse. It reflects the development of the study in evaluative semantics of argumentation in the course of my Ph.D. research. This paper will concentrate on a narrower field that of perceptive evaluations, giving though some insight of the latter's interrelation with other kinds of evaluations.

There are many definitions of argumentation used in various fields of knowledge. A number of theories in various disciplines are involved; these theories will be different according to their object of analysis, their aims, and their methods.

Viewed from a rhetorical point of view argumentation can be defined as a process of proving the validity of some thesis by presenting arguments and counterarguments, evaluating them, and creating in the hearers' minds a belief in the truth and acceptability of the thesis. A distinction has been drawn between logical proof and argumentation; for the former does not concern itself with gaining the agreement of the listener (reader), unlike argumentation, which, for that matter, is a very personal affair, oriented at the audience. An Armenian philosopher and logician, G. Brutyan [3], enlarges the notion of argumentation with the idea 
that apart from the abovesaid, in argumentation "there are given reasons for accepting the thesis with an aim to work out an active social position and carry out certain programs and actions that follow from it".

The same perspective is employed in the definition by Encyclopedia Britannica: "Argumentation, whether it be called rhetorical or dialectical, always aims at persuading or convincing the audience to whom it is addressed of the value of the theses for which it seeks assent". But there is more to argumentation than the usual logical and rhetoric views, observed for hundreds of years. But it stands to reason that argumentative impact and the success of an act of argumentation depend both on the correctness of the discourse as seen from the point of view of rhetoric laws (the order in which the thesis, arguments and examples are introduced), as well as on the semantics of the linguistic units used in the thesis and arguments. A relatively new approach deals with the question of what argumentation, like all human verbal interaction, is built on, i.e. the language and the laws governing its functioning.

From this point of view, argumentation is a complex of linguistic means used for influencing humans' behavior (i.e. as a factor causing to accept this or that decision) as well as a special type of discourse (alongside description, narration, teaching, analysis, etc.). Such a discourse may be characterized by special communicative and illocutionary aims.

From the point of view of semiotics argumentation may be viewed as a particular sort of communication having a certain effect on the addressee's mind, which is organized by the producer of the speech in accordance with the accepted norms of argumentative discourse. Thus, from the point of view of linguistic structures argumentation is seen as functioning of certain elements of the language; from the point of view of the cognitive approach the linguistic study of argumentation is a part of the investigation into the mental models and communicational models of humans.

A person hearing about an event or a thing in evaluative terms is bound to produce his own attitude to the discussed thing - agreeing with the speaker's assessment or not. If a person accepts a given thesis, he evaluates the latter as having a certain value to him. An opponent must be presented solid evidence in support of the virtues of the discussed point both logically and linguistically, so the person giving arguments may in his turn use some evaluative lexical units to subtly lead the hearer towards the positive assessment in favour of the point being proven. In other words, it is possible to suggest evaluative ideas in the linguistic composition of an act of argumentation.

\section{The Role of Evaluative Factor in Argumentation}

An argumentative appeal to the evaluative factors is one of most powerful means of argumentation in natural language. Evaluations possess an argumentative charge themselves in the sense that to assess a thing is to make a decision about it. Therefore, the main purpose of evaluation may be viewed as not to report about facts, but to make an influence. An evaluative attitude is in itself a 
pragmalinguistic category, which influences the semantic structure of the lexical meaning of words used in argumentative discourse. 'Recommendational' power of evaluation, especially addressing emotiveness, is an argument.

The main purpose of evaluative judgements is not to report about facts but to influence people. In the light of the abovesaid my work argues that one of the most influential means of persuading (and in this sense, persuading subconsciously) is the appeal to assessing things and events from the point of view of evaluations based on notions of sense perception - the initial human way of knowing the world. It should be interesting to analyse the work of evaluative meanings of lexical units which ground their semantic bases in various perceptional sensations.

In other words, we are trying to prove the statement that linguistic evaluations possess argumentative meanings. In the simplest terms this might be presented in the following way: "by default" in most usual situations and pictures of the world pairs like "bright - dark", "warm - cold", etc. represent in their first elements more generally accepted positive notions. Thus, if ideas that possess a positive charge in them are used in describing a point, they are likely to create a positive aura in the attitude to this point in the mind of the recipient. In any case, the subtler the evaluation is, the more likely it is to have an effect of agreement in the listener, proving to him the sound basis for the virtues of the thing in question.

I am grounding my categories of evaluations on the classification by a Russian linguist N.D.Arutyunova [1]. Evaluations - with the degree of subtlety (and argumentative power) ascending - are: perceptive or hedonistic; psychological (intellectual and emotive); aesthetic; ethical; utilitarian; normative; and finally teleological. Evaluations having a higher level of sublimity appeal to rational and humanitarian standards in people creating thus a powerful means of influence, making people agree with just and positive features of things as described by the author.

My task here is to see how evaluations based on sense perception come to possess features of higher levels - ethical and aesthetical values. In order to understand the way this mechanism works it is necessary to take into consideration three aspects: qualities of the basic meaning of the word; qualities of the new meaning, i.e. various evaluative meanings; and the connection between the basic and the final meanings. In the latter we see the moment of transition from a descriptive field to evaluative one, reconstructing the semantic development which describes the quality in focus at such a transition. In simple terms - why the idea of "coldness" in the assessment of somebody's behavior is ethically less praised than that of "warmness".

According to a well-known theory of Ch. Stevenson [7], language is used by humans descriptively and dynamically. In the realm of the first usage people's knowledge and views are stored, processed, enriched, distributed among people and passed on to other generations. In case of the dynamic usage people give way to feelings, form beliefs and attitudes toward events, and, most importantly for our case, accept motives for performing certain actions. 
The same view is held by an English researcher, I.A. Richards [6], who names the functions of the language as referential (a mere statement about an event, when words are used for pointing at the referent) and emotive (used for expressing feelings and attitudes to things and events, as well as making others feel and think the same way). In other schools of linguistics these two functions of language are called illocutional and perlocutional respectively.

The pragmatic aim of argumentative acts belongs to the dynamic (emotive or perloctive) function of the language. As was mentioned, it is understood as making a specific influence on the addressee's mind with the help of linguistic means built up according to the accepted in the given culture argumentative principles. The result which the speaker tries to achieve while carrying out such speech acts is to persuade his opponent by verbal means, influence his choice of picking alternatives in the process of making decisions, and, as a result, conduct his behavior in certain ways.

When speaking about axiological (evaluative) meanings of lexical units it is necessary to mention the fact that besides words carrying direct evaluative meanings, there exist such descriptive lexical units which are not characterized in their dictionary definitions as possessing any kind of evaluation. Nevertheless, despite the initial "descriptiveness" of their usage, the latter may be filled with evaluative meanings depending on the context, which, in its turn, leads to their pragmatic functioning, and in particular, to argumentative meanings.

As a tentative analysis based on such a perception of argumentative discourse I would like to offer the following. For the analysis I chose rather a specific text: it is a piece of forensic discourse. Even though it naturally dictates a certain array of rules governing this very type of speech communication, it still uses English with the general realia true to this language. My aim here is not to trace the peculiarities of forensic language within the English verbal communication; rather it is to outline the features true to the language in general. In further research I am intending to use materials from various speech styles. This will reveal what similar features are employed by the language as a representation of a particular culture. The analysis does not claim to be reflecting the state of things in the language with total precision; this is the beginning of a search for patterns of evaluative meanings in the mentality of a national culture.

\section{A Sample Analysis}

The analysis is based on the transcripts of edited and narrated oral court arguments in Roe v. Wade argued on December 13, 1971 in Texas, USA (Edited Supreme Court Opinions Roe v. Wade in [4, pp. 23-45]). Arguments for and against abortion on the territory of the United States of America represent heated debates due to the fact that the subject itself raises highly subjective feelings of both the supporters and antagonists on the issue of abortion, which in its turn is reflected in the character of the speeches.

The aim of this analysis is to try to determine the connectives of evaluative judgements with their perceptive basements in acts of argumentation, and the 
role the latter play in influencing recipient's process of decision-making.

A.N. Baranov in his work on the argumentative culture [2] notes that "lexical meanings of many words that are not even oriented at argumentative dialog, at the same time are not at all strange to argumentative meanings". For example Baranov shows that the most usual procedure for introducing a thesis is "employing an explicative modus with verbs of expressing opinion (in the first person singular, present indefinite tense): suppose, think, consider, etc. Such words introduce proposition, the truth of which it is necessary to prove". A rhetoric aim here is to show one's awareness of the problem and offer the interlocutors to discuss the truth of the claims. By these means it is also shown that the speaker offers a subjective opinion (i.e. anticipates a possible criticism of his point).

In the analysed text the theses are introduced by verbal constructions in the first person (sg. and pl.) as well as by infinitive phrases with the semantic basis on moduses of visual perception and tactile feelings, and of something which we suggest calling "an inner feeling". The latter is especially true in case of women, which can be viewed as the tendency of women to appeal to intuition (a certain "sixth sense") with further use of emotionally colored evaluations in their acts of argumentation.

When introducing a thesis, people more than often use words dealing with visual perception. Almost all of them are synonymous to the moduses of explicative opinion mentioned by Baranov: as I see it, as we see it. Descriptive in their initial meaning, these lexical units introduce the proposition, with an implicit idea that it is a subjective opinion, inviting the opponent to observe it from his own point of view, and (hopefully) accept as true.

Next group of visual moduses concern themselves, on the other hand, with setting objective backgrounds for the offered thesis, they try to point out the real state of things in the world: in view of the fact that, in the light of:

In view of all this, we do not agree that, by adopting one theory of life, Texas may override the rights of the pregnant woman that are at stake.

With respect to the State's important and legitimate interest in the health of the mother, the "compelling" point, in the light of present medical knowledge, is at approximately the end of the first trimester.

Therefore, an opponent is forced to make a conclusion based on a self-evident thesis with which he initially is bound to agree, as provided by the speaker. The word apparent has a rather strong argumentative appeal, and it prompts the listener not to doubt the truth of the claim; thus, it is an objective-based evaluation:

The detriment that the State would impose upon the pregnant woman by denying this choice altogether is apparent. Specific and direct harm medically diagnosable even in early pregnancy may be involved.

The word insight (for such insights as the history may afford us), in the meaning "example" (in-sights) tells us that the review of facts from the past will open to us new perspectives for deciding on the main issue, therefore justifying the necessity of a historical overview and making us pay a special attention to the offered facts: 
Before addressing this claim, we feel it desirable to briefly survey, in several aspects, the history of abortion, for such insight as that history may afford us, and then to examine the state purposes and interests behind the criminal abortion laws.

The expression to color one's thinking and conclusions has a clearly evaluative motivation behind it, though a certain vagueness as to whether the expression has a negative or positive connotation makes this evaluation ambivalent (the immediate understanding of the phrase can vary from "influence $=$ pressure" to "make picturesque"):

One's philosophy, one's experiences, one's exposure to the raw edges of human existence, one's religious training, one's attitudes towards life and family and their values, and the moral standards one establishes and seeks to observe, are all likely to influence and to color one's thinking and conclusions about abortion.

An adequate understanding of this lexical unit is possible only in the context. In this case the expression reflects the difficulty of the abortion problem and the validity of the reasons given by the supporters on the both sides. Therefore, the speaker calls on the participants to give the problem some serious attention. The same is true of the phrase vigorous opposite views:

We forthwith acknowledge our awareness of the sensitive and emotional nature of the abortion controversy, of the vigorous opposite views, even among physicians, and of the deep and seemingly absolute convictions that the subject inspires.

In these two instances the modus of visual perception is a basis for intellectual evaluation (according to the classification by N.D. Arutyunova).

The following quotation may serve as an example of an unsuccessful argumentative act:

Mr. Chief Justice, and may it please the Court. It's an old joke, but when a man argues against two beautiful ladies like this, they are going to have the last word.

(Narrator: No one laughed. Chief Justice Burger looked annoyed. After an embarraced silence, Jay Floyd argued that the case was moot because Jane Roe was no longer pregnant.)

Texas Assistant Attorney General Jay Floyd began his argumentation with a joke that "when a man argues against two beautiful ladies like this, they are going to have the last word". The aesthetic evaluation based on visual perception which is used here is by itself a positive one, but it violated one of Grice's maxims (relevance of the information used), especially being incongruous when used in the court room during important hearings. (This is also shown by the narrator's remarks.)

A.N.Baranov showed that in many cases an appeal to the listener is loaded with some precursory qualities: epistemic (reflecting the speaker's assessment of the possibilities for the alternatives) and axiologic (showing the degree of desirability of this or that answer of the opponent). From this perspective such examples as as I see it and as we see it may be characterized as epistemic, for they just rep- 
resent one of the points of view on this issue, giving the interlocutor freedom to make his own judgements. Other observed examples have axiologic backgrounds because they forward addressees' evaluations on to certain routes.

Further use of evaluative judgements based on visual perception (arguments, counterarguments, examples, conclusions) are grounded on the same principles. For instance, while interpreting The Constitution as having certain rights, it was mentioned that

...these decisions make it clear that not only personal rights that can be deemed "fundamental"... are included in this guarantee of personal privacy. They also make it clear that the right has some extension to activities relating to marriage, ...procreation, ... contraception, ...family relations, .... and child rearing and education.

The repetition of the phrase make it clear states an unarguable truth of such information and is the basis for normative (rational) evaluation.

In the course of the pro-choice supporters' arguments it was stated that the laws of Texas are unconstitutionally vague, thus giving the basis for negative normative and utilitarian (rational) evaluations:

Roe alleged that she was unmarried and pregnant; that she wished to terminate her pregnancy by an abortion "performed by a competent, licensed physician, under safe, clinical conditions"; that she was unable to get a "legal" abortion in Texas because her life did not appear to be threatened by the continuation of her pregnancy; and that she could not afford to travel to another jurisdiction to in order to secure a legal abortion under safe conditions. She claimed that the Texas statutes were unconstitutionally vague and that they abridged her right of personal privacy, protected by the First, Fourth, Fifth, Ninth, and Fourteenth Amendments.

Truly, a law cannot have such a characteristic, and this remark calls for attention in order to clear things out and make the law understandable.

Same aims as the modus of visual perception are served by haptic (tactile) perception; it can be the basis for intellectual and normative evaluations. When speaking about the difficulties of the discussed issue and about the variety of the points of view, someone used an expression one's exposure to the raw edges of human existence, in which the metaphor for the hardships of life is based on the emotionally-utilitarian evaluation expressed by the phrase raw edges:

One's philosophy, one's experiences, one's exposure to the raw edges of human existence, one's religious training, one's attitudes towards life and family and their values, and the moral standards one establishes and seeks to observe, are all likely to influence and to color one's thinking and conclusions about abortion.

It was found that in the analysed text emotional evaluations are very often built on tactile perception. As other examples of emotionally colored evaluations the following may serve: aesthetic evaluation in deformed or defective child, a repetition of the phrase she has no relief, which also carries negative hedonistic evaluation:

If the pregnancy would result in the birth of a deformed or defective child, she has no relief. Regardless of the circumstances of conception, whether it was because of rape, incest, whether she is extremely immature, she has no relief. 
and also the verb to disrupt as referred to life, body, education, family life and work:

I think it's without question that pregnancy to a woman can completely disrupt her life. It disrupts her body; it disrupts her education; it disrupts her employment; and it often disrupts her entire family life.

All of the examples mentioned above belong to women, and this supports the idea that women have a tendency to influence interlocutors more with the help of emotionally colored arguments rather than merely rational ones. Of course such a conclusion should not follow solely from the presented here relatively insufficient number of examples; but it does serve as proof for conclusions about gender differences in verbal communication drawn by a number of linguists, among whom is D. Tannen [8]. Therefore, the meanings of evaluative nouns also possess a certain situational aspect, which is defined as additional information about conditions and participants of the communication process.

For the speech of males, at least in the analysed text, a very characteristic feature is the use of the so-called war metaphors. It concerns teleologic evaluations based on tactile perception (important impact, thrust of an attack), when speaking about the effectiveness of some actions:

The principal thrust of appellant's attack on the Texas statutes is that they improperly invade a right, said to be possessed by the pregnant woman, to chose to terminate her pregnancy.

The original bases for these metaphors (along with the word "invade") are completely obscure now, and for the most part they are widely used without referring to "struggle" or "fight", though the choice of these very phrases has an argumentative aim to make a special impact on the ones who make decisions in this situation.

We called the "inner sense" mentioned above as the basis for a number of evaluations that for the most part (but not always!) characterize the speech of women. Here we include purely emotional evaluations (i.e. emotional by themselves, without context influencing), psychological evaluations and those, based on intuition ("sixth sense"). In the latter category we may include the normative evaluations "emotional response", "feel that", "feel it desirable":

a. Obviously, I have a much more difficult time saying that the state has no interest in late pregnancy.

- Why? Why is that?

- I think it is more the emotional response to late pregnancy, rather than any constitutional...

- Emotional response by whom?

- I guess by persons considering the issue outside the legal context. The Constitution, as I see it, gives protection to people after birth.

b. Before addressing this claim, we feel it desirable to briefly survey, in several aspects, the history of abortion, for such insight as that history may afford us, and then to examine the state purposes and interests behind the criminal abortion laws. 
which provide ground for utilitarian evaluations from the point of view of subjective opinion (in this case these are the synonyms for the observed above subjective evaluations based on visual perception as $I /$ we see it).

Besides, such phrases as "troubling question, passionate argument, sensitive and emotional nature, vigorous opposite views, unsettled and unsettling issue, novel and shocking opinion, distress, distressful life and future" appeal to emotional and hedonistic evaluations based on inner feelings of humans:

a. The Court's opinion brings to the decision of this troubling question both extensive and historical fact and a wealth of legal scholarship.

b. Since then, Americans have spent thousands of hours in passionate argument on this issue.

c. We forthwith acknowledge our awareness of the sensitive and emotional nature of the abortion controversy, of the vigorous opposite views, even among physicians, and of the deep and seemingly absolute convictions that the subject inspires.

d. Chief Justice Warren Burger has called a case that raises an unsettled and unsettling issue in American society, abortion.

e. [The Constitution] is made for people of fundamentally differing views, and the accident of our finding certain opinions natural and familiar or novel and even shocking ought not to conclude our judgment upon the question whether statutes embodying them conflict with the Constitution of the United States.

f. Maternity, or additional offspring, may force upon the woman a distressful life and future. Psychological harm may be imminent. ... There is also the distress, for all concerned, associated with the unwanted child, and there is the problem of bringing a child into a family, already unable, psychologically and otherwise, to care for it.

Such evaluations are motivated by psychological experiences that do not depend on the man's will or self-control; N.D. Arutyunova 11 called these "the most individualized kind of evaluations". Such evaluations orient a person to accommodation in natural and social environments and to reaching comfort. In argumentation all this produces a subjective influence on the listener, launching his own mechanism of sense perception, and in its turn influencing his process of making decisions (axiological condition in decision-making).

The last analysed example with sense perception and hedonistic evaluations showed that such evaluations may house further semantic bases for sublimated and rational evaluations.

The idea of sublimated evaluations is rather new and is described in a number of works of Russian linguists. Sublimated evaluations (from Latin sublimare - elate, exalt, pinnacle) are also called humanised, for they are placed above evaluations based on sense-perception, "humanising" them. [5]. According to N.D. Arutyunova [1] "this type of evaluations is closely connected with the term archetype, i.e. norm, example, potential requirements set towards the object". Rational evaluations, on the other hand, "are connected with practical activities, practical interests and everyday experiences of people. Their basic criteria is physical and psychological good, oriented at reaching a certain goal, performing a certain function, meeting certain standards" [5].

Thus, the above mentioned evaluations due to their argumentative influence, 
besides the immediate impact on the interlocutors through their direct senseperception evaluative meanings, acquire additional meanings, which reveal in the topics under consideration new ethical and humanistic values.

"We face re-orientation of sense-perception, psychological and rational evaluations towards spiritual values, so that in their turn these evaluations serve as semantic bases for sublimated evaluations" 5 . Trying to prove to the opponents the truth of their positions, speakers resort to these complicated evaluative constructions appealing to the listeners' normative and ethical ideals.

Besides, among major conditions for choosing evaluative lexical units in arguments, there exist other factors such as social role-status of interlocutors, their emotional state at the moment of speaking, and context.

In the court room role statuses are very well marked and the possible choice of speech acts is narrowed to restrained and rather high-flown style; evaluations in their turn are restricted to barely emotional. Therefore in the text we did not find explicitly expressed emotional, ethical, aesthetical, teleological, normative and utilitarian evaluations. Such types of evaluations are found only as based on other (sense perception and hedonistic) evaluations. Emotional state of interlocutors and verbal context are defined by a wider, extralinguistic context, i.e. corresponding to the requirements of a court session. Emotiveness finds vent through the use of moduses of visual, tentative and inner-sense perception. The context circumscribes the use of the language by resorting to relevant information only, without unnecessary emotiveness and unrelated sophistic casistry.

\section{References}

1. Arutyunova N.D. ed. Logichesky analiz yazyka: yazyki dinamicheskogo mira (Logical Analysis of the Language: Language of the Dynamic World), Dubna, 1999.

2. Baranov A.N., Sergeev V.M. Estestvenno-yazykovaya argumentatsiya v logike prakticheskogo rassuzhdeniya (Natural-Language Argumentation in the Logics of Practical Reasoning). In: Myshlenie, kognitivnye nauki, intellekt (Thinking, Cognitive Sciences and Artificial Intelligence), Moskva, 1988. pp. 104-119.

3. Brutyan G.A., Argumentatsiya (Argumentation). Yerevan, 1984.

4. Gutton, Stephanie and Peter Irons, ed. May It Please the Court: Arguments on Abortion. The New Press, NY, 1995.

5. Pisanova T.V. Natsional'no-kul'turnye aspekty otsenochnoi semantiki: eticheskie $i$ esteticheskie otsenki (National and Cultural Aspects of Evaluative Semantics: Aesthetical and Ethical Evaluations). - Moskva, 1997.

6. Richards I.A. \& Ogden C.K. The Meaning of Meaning: A Study of the Influence of Language upon Thought and the Science of Symbolism. New York: Harcourt, Brace, 1930.

7. Stevenson Ch.L. Facts and Values: Studies in Ethical Analysis. - New Haven London: Yale Univ. Press, 1964.

8. Tannen D. The Argument Culture. Stopping American War of Words. The Ballantine Publishing Group, NY, 1998. 